MATHEMATICS OF COMPUTATION

Volume 73, Number 246, Pages 517-523

S $0025-5718(03) 01570-9$

Article electronically published on June 17, 2003

\title{
ASYMPTOTICALLY EXACT A POSTERIORI ESTIMATORS FOR THE POINTWISE GRADIENT ERROR ON EACH ELEMENT IN IRREGULAR MESHES. PART II: THE PIECEWISE LINEAR CASE
}

\author{
ALFRED H. SCHATZ AND LARS B. WAHLBIN
}

\begin{abstract}
We extend results from Part I about estimating gradient errors elementwise a posteriori, given there for quadratic and higher elements, to the piecewise linear case. The key to our new result is to consider certain technical estimates for differences in the error, $e\left(x_{1}\right)-e\left(x_{2}\right)$, rather than for $e(x)$ itself. We also give a posteriori estimators for second derivatives on each element.
\end{abstract}

\section{INTRODUCTION}

As in Part I, [3], we consider a second order elliptic partial differential equation with a natural homogeneous Neumann conormal boundary condition. Let $\Omega$ be a bounded domain in $R^{N}$ with a smooth boundary and, for simplicity of presentation at certain points in our present arguments, we now assume it is also convex. The bilinear form on $W_{2}^{1}(\Omega)$ associated with the partial differential equation,

$$
A(v, w)=\int_{\Omega}\left(\sum_{i, j=1}^{N} a_{i j}(x) \frac{\partial v}{\partial x_{i}} \frac{\partial w}{\partial x_{j}}+\sum_{i=1}^{N} b_{i}(x) \frac{\partial v}{\partial x_{i}} w+c(x) v w\right) d x
$$

is assumed to have smooth coefficients on $\bar{\Omega}$ and, again for simplicity of presentation, to be coercive. I.e., there is $c_{\text {coer }}>0$ such that $c_{\text {coer }}\|v\|_{W_{2}^{1}(\Omega)}^{2} \leq A(v, v)$, for all $v \in$ $W_{2}^{1}(\Omega)$.

Now consider approximation of the solution $u$ to the problem $A(u, \varphi)=(f, \varphi) \equiv$ $\int_{\Omega} f \varphi d x$, for all $\varphi \in W_{2}^{1}(\Omega)$. For $0<h<1$, let $S_{h}$ be the subspace of $W_{2}^{1}(\Omega)$ consisting of continuous piecewise linear functions defined on globally quasi-uniform and globally shape-regular simplicial triangulations of $\Omega$ that fit $\partial \Omega$ exactly. Thus, elements with curved faces are allowed at the boundary. Let $u_{h} \in S_{h}$ be the standard Galerkin finite element approximation of $u$ defined by $A\left(u_{h}, \varphi\right)=(f, \varphi)$, for all $\varphi \in S_{h}$, so that

$$
A\left(u-u_{h}, \varphi\right)=0, \quad \text { for all } \varphi \in S_{h} .
$$

Our primary aim is to study asymptotically exact a posteriori estimators for $\|\nabla e\|_{L_{\infty}(\tau)}, e=u-u_{h}$, the maximum norm of the gradient error on any given

Received by the editor April 12, 2002 and, in revised form, September 7, 2002.

2000 Mathematics Subject Classification. Primary 65N30, 65N15.

Both authors were supported by the National Science Foundation, USA, Grant DMS-0071412. They thank a referee for suggesting improvements in the presentation. 
element. The problem of estimating second derivatives of $u$ will also be studied. Our estimators for the gradient error will be of the form

$$
\mathcal{E}(\tau)=\left\|\nabla u_{h}-\mathcal{G}_{H} u_{h}\right\|_{L_{\infty}(\tau)},
$$

where $\mathcal{G}_{H} v$ is an averaging operator that will be defined in terms of a domain $d_{H}$ which includes $\tau$ and is of diameter $H$, for some $H \geq 2 h$. We shall assume that $\mathcal{G}_{H}$ has the following properties:

$$
\mathcal{G}_{H} 1=0 \text {, and }\left\|\nabla v-\mathcal{G}_{H} v\right\|_{L_{\infty}(\tau)} \leq C_{\mathcal{G}} H^{2}\|v\|_{W_{\infty}^{3}\left(d_{H}\right)} \text {, for } v \in C^{3}\left(\bar{d}_{H}\right),
$$

and

$$
\left\|\mathcal{G}_{H} v\right\|_{L_{\infty}(\tau)} \leq C_{\mathcal{G}} H^{-1}\|v\|_{L_{\infty}\left(d_{H}\right)}, \quad \text { for } v \in C\left(\bar{d}_{H}\right) .
$$

The inequality in (1.3) says that $\mathcal{G}_{H} v$ is locally a second order (in $H$ ) approximation to the gradient, and (1.4) may be interpreted as a smoothing property. We note that, for a given $d_{H}$, any element $\tau$ in it will work. I.e., it is not necessary to change $d_{H}$ for each and every $\tau$. We shall give three examples of operators satisfying these properties. The verification that they hold is essentially given in [3].

Example 1. Let $d_{H} \subseteq \Omega$ be such that $d_{H}$ contains a ball $\underline{B}$ of radius $\underline{C}_{1} H, \underline{C}_{1}>0$ and is contained in a concentric ball $\bar{B}$ of radius $\bar{C}_{1} H$, and where meas $\left(\partial d_{H}\right) \leq$ $\bar{C}_{1} H^{N-1}$. In particular $d_{H}$ could be a mesh domain. Let $\Pi_{1}\left(d_{H}\right)$ be the space of first degree, affine polynomials restricted to $\bar{d}_{H}$. We define $\mathcal{G}_{H} v=P_{H}^{1} \nabla v$, where $P_{H}^{1}$ is the componentwise $L_{2}$-projection into $\Pi_{1}\left(d_{H}\right)$.

Example 2. Let $d_{H}$ be as in Example 1 let $\Pi_{2}\left(d_{H}\right)$ be the quadratic polynomials, and let $\mathcal{G}_{H} v=\nabla P_{H}^{2} v$. In this example we could replace the $L_{2}$-projection $P_{H}^{2}$ by a suitable approximation, such as interpolating $v$ into $\Pi_{2}\left(d_{H}\right)$ at $N^{2} / 2+3 N / 2+1$ appropriately placed points or using a discrete $L_{2}$-projection at a greater number of points.

Example 3. For each $x \in \tau$, let $\mathcal{G}_{H} v(x)=\left(Q_{1}^{H} v(x), \ldots, Q_{N}^{H} v(x)\right)$, where each $Q_{i}^{H}$ is a second order accurate difference approximation to $\frac{\partial}{\partial x_{i}}$. If $\operatorname{dist}(\tau, \partial \Omega) \geq \bar{C}_{2} H$, then we may take each

$$
Q_{i}^{H} v(x)=\frac{v\left(x+H e_{i}\right)-v\left(x-H e_{i}\right)}{2 H},
$$

the standard second order accurate centered difference approximation to $\frac{\partial v}{\partial x_{i}}$. Here, $e_{i}$ is the unit vector in the positive $x_{i}$ direction. Near the boundary, one-sided differences may be employed, but we shall not give details.

Our main result, which is an extension of Theorem 2.1 of [3] to the piecewise linear case, is as follows.

Theorem 1.1. Fix $0<\varepsilon<1$. Let $\mathcal{G}_{H}$ satisfy (1.3) and (1.4). There exists a constant $C_{1}$ such that with

$$
m:=C_{1}\left(\left(\frac{H}{h}\right)^{2} h^{\varepsilon}+\left(\frac{h}{H}\right)^{\varepsilon} \ln \left(\frac{H}{h}\right)\right),
$$

and $u$ and $u_{h} \in S_{h}$ satisfying (1.1), one of the following two alternatives holds for each element $\tau$. 
Alternative I. Suppose that on the element $\tau$, the function $u$ satisfies

$$
|u|_{W_{\infty}^{2}(\tau)} \geq h^{1-\varepsilon}\|u\|_{W_{\infty}^{3}(\Omega)} .
$$

In this case

$$
\left\|\nabla u-\mathcal{G}_{H} u_{h}\right\|_{L_{\infty}(\tau)} \leq m\|\nabla e\|_{L_{\infty}(\tau)} .
$$

If $H=H(h)$ is chosen so that $m<1$, then our estimator given in (1.2) is equivalent to the real gradient error on the element,

$$
\frac{1}{1+m} \mathcal{E}(\tau) \leq\|\nabla e\|_{L_{\infty}(\tau)} \leq \frac{1}{1-m} \mathcal{E}(\tau)
$$

Furthermore, if $H(h)$ is chosen so that $m \rightarrow 0$ as $h \rightarrow 0$, our estimator is asymptotically exact for the gradient error on the element.

Alternative II. Suppose that (1.5) does not hold, i.e.,

$$
|u|_{W_{\infty}^{2}(\tau)}<h^{1-\varepsilon}\|u\|_{W_{\infty}^{3}(\Omega)} .
$$

In this case $\|\nabla e\|_{L_{\infty}(\tau)}$ is "small" with

$$
\|\nabla e\|_{L_{\infty}(\tau)} \leq C_{1} h^{2-\varepsilon}\|u\|_{W_{\infty}^{3}(\Omega)},
$$

and our error indicator is similarly "small",

$$
\mathcal{E}(\tau) \leq\left(C_{1}+m\right) h^{2-\varepsilon}\|u\|_{W_{\infty}^{3}(\Omega)} .
$$

In the above, $C_{1}$ depends on $N, \Omega, c_{c o e r}, a_{i j}, b_{i}, c$, constants of quasi-uniformity and shape-regularity for the meshes, $C_{\mathcal{G}}$, and $\varepsilon$.

Remark 1.1. In the case that $\mathcal{G}_{H} u_{h}$ gives an asymptotically exact estimator for $\nabla u$, it is a better approximation to $\nabla u$ than $\nabla u_{h}$ is.

Remark 1.2. For a discussion of how results of this type relate to the previous literature on a posteriori estimates, and for a fuller description of the general framework of the methods considered here, see Part I [3].

Remark 1.3. Here we shall make two comments that may give some insight into the role that condition (1.5) plays towards insuring that the locally defined estimator $\mathcal{E}(\tau)$ is asymptotically exact. First of all, it follows from (1.5), and Lemmas 2.1 and 2.4 below that, for $h$ sufficiently small,

$$
C_{*} h|u|_{W_{\infty}^{2}(\tau)} \leq\|\nabla e\|_{L_{\infty}(\tau)} \leq C^{*} h|u|_{W_{\infty}^{2}(\tau)} .
$$

This says that the finite element gradient error on $\tau$ has a similar type of local behavior as the interpolation error. So it is plausible that a locally defined estimator may be effective. Secondly, asymptotic exactness follows if we can show that, roughly speaking, $\mathcal{G}_{H} u_{h}$ is a better approximation to $\nabla u$ than is $\nabla u_{h}$. Now condition (1.3) says that the best that we can expect even $\mathcal{G}_{H} u$ to approximate $\nabla u$ is $O\left(H^{2}\right)$, or roughly $O\left(h^{2}\right)$ (since we roughly want $H$ to be only slightly larger than $h)$. The "worst" case of condition (1.5) occurs when $|u|_{W_{\infty}^{2}(\tau)}=h^{1-\varepsilon}\|u\|_{W_{\infty}^{3}(\Omega)}$. Combining this with the estimate above, we see that

$$
\|\nabla e\|_{L_{\infty}(\tau)} \leq C h^{2-\varepsilon}\|u\|_{W_{\infty}^{3}(\Omega)} .
$$

Thus, at least for $\varepsilon$ small, we are at a point past which we have no reason to expect that $\mathcal{G}_{H} u_{h}$ would be much closer to $\nabla u$ than $\nabla u_{h}$ is. 
In general, if (1.5) is violated, it may happen that $|u|_{W_{\infty}^{2}(\tau)} \leq h\|u\|_{W_{\infty}^{3}(\Omega)}$. In such a situation, Lemma 2.1 actually gives

$$
\|\nabla e\|_{L_{\infty}(\tau)} \leq C^{\prime} h^{2-\varepsilon^{\prime}}\|u\|_{W_{\infty}^{3}(\Omega)},
$$

for any $\varepsilon^{\prime}>0$. In this case, surely we have no reason to expect that $\mathcal{G}_{H} u_{h}$ would be a much better approximation.

We now turn to estimates for the second derivatives of $u$ on an element $\tau$. Of course, since the second derivatives of the piecewise linear function $u_{h}$ are zero (the second derivatives being regarded in an elementwise fashion), here we are not speaking about estimating errors, but merely about the size itself of second derivatives of $u$. Let $D^{\beta} u,|\beta|=2$, denote any second order derivative, and let $\mathcal{G}_{H}^{(\beta)} u_{h}$ denote the analogue coming from taking a derivative, elementwise, of a component of $\mathcal{G}_{H} u_{h}$. (For the mixed derivatives, two choices are possible.)

To be precise, let $|u|_{W_{\infty}^{2}(\tau)}=\max _{|\beta|=2}\left\|D^{\beta} u\right\|_{L_{\infty}(\tau)}$, and similarly let

$$
\mathcal{E}^{(2)}(\tau)=\max _{|\beta|=2}\left\|\mathcal{G}_{H}^{(\beta)} u_{h}\right\|_{L_{\infty}(\tau)}
$$

We assume that

$$
\left\|\nabla v-\mathcal{G}_{H} v\right\|_{W_{\infty}^{1}(\tau)} \leq C_{\mathcal{G}} H\|v\|_{W_{\infty}^{3}\left(d_{H}\right)}, \quad \text { for } v \in C^{3}\left(\bar{d}_{H}\right),
$$

and

$$
\left\|\nabla \mathcal{G}_{H} v\right\|_{L_{\infty}(\tau)} \leq C_{\mathcal{G}} H^{-1}\|v\|_{W_{\infty}^{1}\left(d_{H}\right)}, \quad \text { for } v \in W_{\infty}^{1}\left(\bar{d}_{H}\right) .
$$

It is easy to check that the operators in Examples 103 satisfy (1.12) and (1.13). (The verification of (1.13) in the case of Example 2 uses that $\mathcal{G}_{H} 1=0$.)

We now have:

Theorem 1.2. Fix $0<\varepsilon<1$. Assume that (1.12) and (1.13) hold. There exists a constant $C_{2}$ such that with

$$
\widetilde{m}:=C_{2}\left(\left(\frac{H}{h}\right) h^{\varepsilon}+\left(\frac{h}{H}\right)^{\varepsilon}\right)
$$

and $u$ and $u_{h}$ satisfying (1.1), one of the following two alternatives holds for each element $\tau$.

Alternative I. Suppose that (1.5) holds. In this case,

$$
\left\|D^{\beta} u-\mathcal{G}_{H}^{(\beta)} u_{h}\right\|_{L_{\infty}(\tau)} \leq \widetilde{m}|u|_{W_{\infty}^{2}(\tau)}, \quad \text { for each }|\beta|=2 .
$$

If $H=H(h)$ is chosen so that $\widetilde{m}<1$, then our estimator given in (1.11) is an equivalent estimator,

$$
\frac{1}{1+\widetilde{m}} \mathcal{E}^{(2)}(\tau) \leq|u|_{W_{\infty}^{2}(\tau)} \leq \frac{1}{1-\widetilde{m}} \mathcal{E}^{(2)}(\tau) .
$$

If $H(h)$ is chosen so that $\widetilde{m} \rightarrow 0$ as $h \rightarrow 0$, then the estimator is asymptotically exact.

Alternative II. Suppose (1.8) holds. Then, of course, $|u|_{W_{\infty}^{2}(\tau)} \leq h^{1-\varepsilon}\|u\|_{W_{\infty}^{3}(\Omega)}$ is already "small". We then assert that our estimator is similarly "small",

$$
\mathcal{E}^{(2)}(\tau) \leq(1+\widetilde{m}) h^{1-\varepsilon}\|u\|_{W_{\infty}^{3}(\Omega)} .
$$


Remark 1.4. For simplicity of presentation, we have only considered estimators for second derivatives of $u$ of the form: take an elementwise derivative of $\mathcal{G}_{H} u_{h}$. Certainly, instead of using straight differentiation, one could use iterated variants of $\mathcal{G}_{H}$, cf., e.g., Eriksson and Johnson [2]. Results similar to Theorem 1.2 are readily derived.

Remark 1.5. In the case that (1.5) holds and $\widetilde{m} \rightarrow 0$ as $h \rightarrow 0$, then (1.14) says that $\mathcal{G}_{H}^{(\beta)} u_{h}$ converges to $D^{\beta} u$ on $\tau$.

An outline of the rest of this note is as follows. In Section 2 we collect two a priori estimates, following Schatz 4 and Schatz and Wahlbin [5, and some other preliminary material. In particular, following Demlow [1, we elucidate why the piecewise linear case was not included in Part I of this paper. In Section 3 we prove Theorems 1.1 and 1.2

\section{SOME PRELIMINARES}

From [4] we have the following asymptotic error expansion inequality.

Lemma 2.1. For any $\varepsilon>0$, there exists a constant $C$ such that

$$
|e(x)|+|\nabla e(x)| \leq C h\left(\max _{|\beta|=2}\left|D^{\beta} u(x)\right|+h^{1-\varepsilon}\|u\|_{W_{\infty}^{3}(\Omega)}\right) .
$$

A key estimate used in [3] was a similar expansion inequality for $e(x)$ alone, proven in [4] for piecewise quadratics or higher order elements. This estimate is of the form

$$
|e(x)| \leq C h^{r}\left(\max _{|\alpha|=r}\left|D^{\alpha} u(x)\right|+h^{1-\varepsilon}\|u\|_{W_{\infty}^{r+1}(\Omega)}\right), \quad \text { for } r \geq 3,
$$

where $r=3$ corresponds to piecewise quadratics, etc. In [1] it has been shown, via a simple example in one space dimension, that such an estimate is impossible in the piecewise linear case, $r=2$. As a substitute, we shall instead use the following recent result from [5].

For $x_{1}, x_{2} \in \Omega$, let $\rho=h+\left|x_{2}-x_{1}\right|$ and $\bar{x}=\left(x_{1}+x_{2}\right) / 2$.

Lemma 2.2. For any $\varepsilon>0$, there exists a constant $C$ such that

$$
\left|e\left(x_{2}\right)-e\left(x_{1}\right)\right| \leq C h^{2}(1+\ln (\rho / h))\left(\max _{|\beta|=2}\left|D^{\beta} u(\bar{x})\right|+\rho^{1-\varepsilon}\|u\|_{W_{\infty}^{3}(\Omega)}\right) .
$$

We next record a trivial fact which however hints at how Lemma 2.2 will come into play.

Lemma 2.3. Let $\mathcal{G}_{H}$ satisfy (1.3) and (1.4). Then for any point $z \in d_{H}$,

$$
\left\|\mathcal{G}_{H} v\right\|_{L_{\infty}(\tau)} \leq \frac{C}{H}\|v(\cdot)-v(z)\|_{L_{\infty}\left(d_{H}\right)} .
$$

Proof. Since $\mathcal{G}_{H} 1=0$, this follows from (1.4).

Finally, essentially from approximation theory, there is a lower bound for gradient errors on an element; see [3, Lemma 3.3], for a proof.

Lemma 2.4. There exists a constant $c>0$ such that

$$
c\left(h|u|_{W_{\infty}^{2}(\tau)}-h^{2}\|u\|_{W_{\infty}^{3}(\tau)}\right) \leq\|\nabla e\|_{L_{\infty}(\tau)} .
$$




\section{Proofs of the theOREMS}

Proof of Theorem 1.1. We have, by use of (1.3) and Lemma 2.3. with any $z \in d_{H}$,

$$
\begin{aligned}
\left\|\nabla u-\mathcal{G}_{H} u_{h}\right\|_{L_{\infty}(\tau)} & \leq\left\|\nabla u-\mathcal{G}_{H} u\right\|_{L_{\infty}(\tau)}+\left\|\mathcal{G}_{H} e\right\|_{L_{\infty}(\tau)} \\
& \leq C H^{2}\|u\|_{W_{\infty}^{3}(\Omega)}+\frac{C}{H}\|e(\cdot)-e(z)\|_{L_{\infty}\left(d_{H}\right)} .
\end{aligned}
$$

Let $x_{0}$ be a point where $\|e(\cdot)-e(z)\|_{L_{\infty}\left(d_{H}\right)}$ is taken on, and let $\bar{x}=\left(x_{0}+z\right) / 2$. Then, by Lemma 2.2 and the mean-value theorem, since $\operatorname{dist}(\bar{x}, \tau) \leq H$,

$$
\begin{aligned}
\|e(\cdot)-e(z)\|_{L_{\infty}\left(d_{H}\right)} & \leq C h^{2}(\ln (H / h))\left(\max _{|\beta|=2}\left|D^{\beta} u(\bar{x})\right|+H^{1-\varepsilon}\|u\|_{W_{\infty}^{3}(\Omega)}\right) \\
& \leq C h^{2}(\ln H / h)\left(|u|_{W_{\infty}^{2}(\tau)}+H^{1-\varepsilon}\|u\|_{W_{\infty}^{3}(\Omega)}\right) .
\end{aligned}
$$

Thus, from (3.1),

$$
\begin{aligned}
\left\|\nabla u-\mathcal{G}_{H} u_{h}\right\|_{L_{\infty}(\tau)} \leq & C H^{2}\|u\|_{W_{\infty}^{3}(\Omega)}+C \frac{h^{2}}{H} \ln (H / h)|u|_{W_{\infty}^{2}(\tau)} \\
& +C \frac{h^{2}}{H^{\varepsilon}} \ln (H / h)\|u\|_{W_{\infty}^{3}(\Omega)} .
\end{aligned}
$$

In case of Alternative I, $\|u\|_{W_{\infty}^{3}(\Omega)} \leq h^{-1+\varepsilon}|u|_{W_{\infty}^{2}(\tau)}$, we hence obtain

$$
\begin{aligned}
\left\|\nabla u-\mathcal{G}_{H} u_{h}\right\|_{L_{\infty}(\tau)} & \\
\leq & C\left(\frac{H^{2}}{h^{1-\varepsilon}}+\frac{h^{2}}{H} \ln (H / h)+\frac{h^{1+\varepsilon}}{H^{\varepsilon}} \ln (H / h)\right)|u|_{W_{\infty}^{2}(\tau)} \\
& \leq C\left(\frac{H^{2}}{h^{1-\varepsilon}}+\frac{h^{1+\varepsilon}}{H^{\varepsilon}} \ln (H / h)\right)|u|_{W_{\infty}^{2}(\tau)} .
\end{aligned}
$$

From Lemma 2.4 in our present Alternative I, for $h$ small, $c h|u|_{W_{\infty}^{2}(\tau)} \leq\|\nabla e\|_{L_{\infty}(\tau)}$, and hence from (3.3),

$$
\left\|\nabla u-\mathcal{G}_{H} u_{h}\right\|_{L_{\infty}(\tau)} \leq C\left(\left(\frac{H}{h}\right)^{2} h^{\varepsilon}+\left(\frac{h}{H}\right)^{\varepsilon} \ln (H / h)\right)\|\nabla e\|_{L_{\infty}(\tau)} .
$$

This is (1.6). Obviously, (1.7) follows from this by the triangle inequality.

In the case of Alternative II, $|u|_{W_{\infty}^{2}(\tau)} \leq h^{1-\varepsilon}\|u\|_{W_{\infty}^{3}(\Omega)}$, we have from Lemma 2.1

$$
\|\nabla e\|_{L_{\infty}(\tau)} \leq C h^{2-\varepsilon}\|u\|_{W_{\infty}^{3}(\Omega)}
$$

which is (1.9). From (3.2) we now get

$$
\begin{aligned}
\| \nabla u & -\nabla \mathcal{G}_{H} u_{h}\left\|_{L_{\infty}(\tau)} \leq C\left(H^{2}+\frac{h^{3-\varepsilon}}{H} \ln (H / h)+\frac{h^{2}}{H^{\varepsilon}} \ln (H / h)\right)\right\| u \|_{W_{\infty}^{3}(\Omega)} \\
& =C\left(\left(\frac{H}{h}\right)^{2} h^{\varepsilon}+\frac{h}{H} \ln (H / h)+\left(\frac{h}{H}\right)^{\varepsilon} \ln (H / h)\right) h^{2-\varepsilon}\|u\|_{W_{\infty}^{3}(\Omega)} \\
& \leq m h^{2-\varepsilon}\|u\|_{W_{\infty}^{3}(\Omega)},
\end{aligned}
$$

and hence (1.10) also follows. This completes the proof of Theorem 1.1 .

Proof of Theorem 1.2. We have, using (1.12) and (1.13),

$$
\begin{aligned}
\left\|D^{\beta} u-\mathcal{G}_{H}^{(\beta)} u_{h}\right\|_{L_{\infty}(\tau)} & \leq\left\|D^{\beta} u-\mathcal{G}_{H}^{(\beta)} u\right\|_{L_{\infty}(\tau)}+\left\|\mathcal{G}_{H}^{(\beta)} e\right\|_{L_{\infty}(\tau)} \\
& \leq C H\|u\|_{W_{\infty}^{3}(\Omega)}+\frac{C}{H}\|e\|_{W_{\infty}^{1}\left(d_{H}\right)} .
\end{aligned}
$$


From Lemma 2.1, using the mean-value theorem, we find that

$$
\|e\|_{W_{\infty}^{1}\left(d_{H}\right)} \leq C h\left(|u|_{W_{\infty}^{2}(\tau)}+H^{1-\varepsilon}\|u\|_{W_{\infty}^{3}(\Omega)}\right) .
$$

Hence, from (3.4),

$$
\left\|D^{\beta} u-\mathcal{G}_{H}^{(\beta)} u_{h}\right\|_{L_{\infty}(\tau)} \leq C H\|u\|_{W_{\infty}^{3}(\Omega)}+C \frac{h}{H}|u|_{W_{\infty}^{2}(\tau)}+C \frac{h}{H^{\varepsilon}}\|u\|_{W_{\infty}^{3}(\Omega)} .
$$

Thus, in case of Alternative I, $\|u\|_{W_{\infty}^{3}(\Omega)} \leq h^{-1+\varepsilon}|u|_{W_{\infty}^{2}(\tau)}$,

$$
\left\|D^{\beta} u-\mathcal{G}_{H}^{(\beta)} u_{h}\right\|_{L_{\infty}(\tau)} \leq \widetilde{m}|u|_{W_{\infty}^{2}(\tau)},
$$

and Theorem 1.2 (1.15) and the asymptotic equivalence, follows in this case.

In Alternative II, $|u|_{W_{\infty}^{2}(\tau)} \leq h^{1-\varepsilon}\|u\|_{W_{\infty}^{3}(\Omega)}$, and (3.5) gives

$$
\begin{aligned}
\left\|D^{\beta} u-\mathcal{G}_{H}^{(\beta)} u_{h}\right\|_{L_{\infty}(\tau)} & \leq C\left(H+\frac{h^{2-\varepsilon}}{H}+\frac{h}{H^{\varepsilon}}\right)\|u\|_{W_{\infty}^{3}(\Omega)} \\
& =C\left(\left(\frac{H}{h}\right) h^{\varepsilon}+\frac{h}{H}+\left(\frac{h}{H}\right)^{\varepsilon}\right) h^{1-\varepsilon}\|u\|_{W_{\infty}^{3}(\Omega)} \\
& \leq \widetilde{m} h^{1-\varepsilon}\|u\|_{W_{\infty}^{3}(\Omega)} .
\end{aligned}
$$

Via the triangle inequality, this proves (1.16) and completes the proof of Theorem 1.2.

\section{REFERENCES}

[1] A. Demlow, Piecewise linear finite elements methods are not localized, Math. Comp. (to appear).

[2] K. Eriksson and C. Johnson, An adaptive finite element method for linear elliptic problems, Math. Comp. 50 (1988), 361-384. MR 89c:65119

[3] W. Hoffmann, A. H. Schatz, L. B. Wahlbin and G. Wittum, Asymptotically exact a posteriori estimators for the pointwise gradient error on each element in irregular meshes. Part I: A smooth problem and globally quasi-uniform meshes, Math. Comp. 70 (2001), 897-909. MR 2002a: 65178

[4] A. H. Schatz, Pointwise error estimates and asymptotic error expansion inequalities for the finite element method on irregular grids: Part I. Global estimates, Math. Comp. 67 (1998), 877-899. MR 98j:65082

[5] A. H. Schatz and L. B. Wahlbin, Pointwise error estimates for differences of piecewise linear finite element approximations, SIAM J. Numer. Anal. (to appear).

Department of Mathematics, Cornell University, Ithaca, New York 14853

E-mail address: schatz@math.cornell.edu

Department of Mathematics, Cornell University, Ithaca, New York 14853

E-mail address: wahlbin@math.cornell.edu 